\title{
Factores de riesgo asociados a la disfunción de una fístula arteriovenosa en pacientes con enfermedad renal crónica
}

\author{
Juan Carlos Sánchez González
}

Enfermero, Fundación Jiménez Díaz, Madrid

\section{Resumen}

La enfermedad renal se ha convertido en una patología crónica de gran prevalencia en los países industrializados. Una modalidad de tratamiento sustitutivo renal es la hemodiálisis, para la cual es imprescindible tener un acceso vascular. Como acceso vascular de elección preferible al catéter venoso central, está la fístula arteriovenosa por haber demostrado menos complicaciones. Sin embargo, no está exenta de riesgos y dado que los problemas relacionados con el acceso vascular constituyen la primera causa de morbilidad y hospitalización, se pretendió estudiar aquellos factores de riesgo que influyen en la disfunción de las fístulas arteriovenosas.

\section{Material y métodos:}

Se trata de un diseño de caso-control en el que se comparó el grupo que presentó alguna disfunción de su fístula, ya fuera autóloga o protésica, con el que no presentó ninguna disfunción para analizar las diferencias entre ambos. Las variables se agruparon en tres apartados definidos para organizar los resultados y se estratificó por

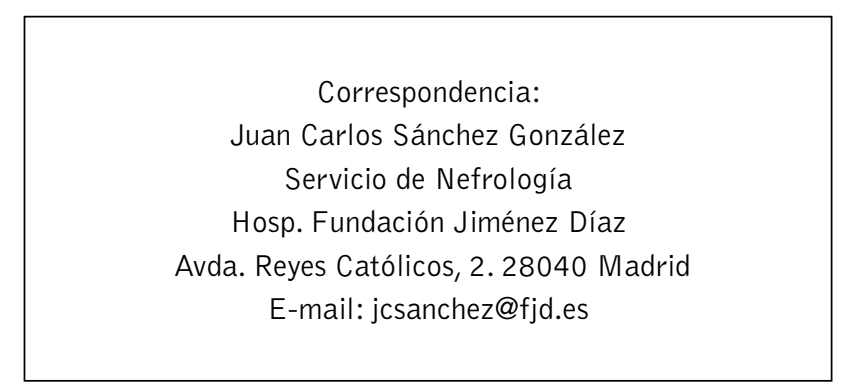

sexo, edad y por haber padecido o no disfunción de la fístula.

\section{Resultados:}

La edad y el sexo no influyen en padecer disfunción de la fístula sino que tiene más influencia la dislipemia, el haber comenzado tratamiento con hemodiálisis, el tipo de fístula y el haber portado catéter venoso central.

\section{Discusión:}

A diferencia de estudios publicados, en nuestra población no se han encontrado diferencias de disfunción de fístula en cuanto a edad y sexo. Sin embargo, se ha visto que hay menos riesgo de disfunción en aquellos pacientes con dislipemia, en los que su fístula es autóloga y en los que no han iniciado hemodiálisis o lo han hecho de manera programada $y$, por tanto, no han portado catéteres venosos centrales.
PALABRAS CLAVE:

- FÍSTULA ARTERIOVENOSA

- ACCESO VASCULAR

- DISFUNCIÓN; FACTOR DE RIESGO

- HEMODIÁLISIS. 
Risk factors associated with a fistula arteriovenous dysfunction in patients with chronic kidney disease

\section{Abstract}

Renal disease has become a chronic disease of high prevalence in industrialized countries. A form of renal replacement therapy is hemodialysis, which is essential to have a vascular access. As vascular access of choice preferable to central venous catheter is arteriovenous fistula for showing fewer complications. However, it is not without risks and problems as related to vascular access are the leading cause of morbidity and hospitalization, we intended to study the risk factors that influencing arteriovenous fistulas dysfunction.

\section{Methods}

This is a case-control design in which we compared the group with some fistula dysfunction, either autologous or prosthetic, with which presented no dysfunction to analyze the differences between both. The variables were grouped into three sections defined to organize the results and stratified by gender, age and having suffered or not fistula dysfunction.

\section{Results}

Age and gender do not influence fistula dysfunction but has more influence dyslipidemia, having started hemodialysis, type of fistula and have carried central venous catheter.

\section{Discussion}

Unlike studies published, in our population did not find fistula dysfunction differences in age and gender. However, it is seen that there is less risk of dysfunction in patients with dyslipidemia, wherein the fistula is autologous and the hemodialysis have not started or have done on a scheduled start and, therefore, they have not carried central venous catheters.

\section{KEYWORDS: \\ - ARTERIOVENOUS FISTULA \\ - ACCESS VASCULAR \\ - DYSFUNCTION \\ - RISK FACTOR \\ - HEMODIALYSIS}

\section{Introducción}

En los países industrializados, en el momento actual, las enfermedades crónicas constituyen una seria amenaza para la salud de la población y las predicciones sanitarias indican que la prevalencia de la enfermedad cardiovascular va a alcanzar niveles epidémicos en todo el mundo en los próximos años, sobre todo debido al aumento de la obesidad y la diabetes en la población general ${ }^{1}$.

A parte de las enfermedades cardiovasculares y oncológicas, la enfermedad renal crónica (ERC) se ha convertido en un problema de salud pública a nivel mundial ${ }^{2}$. Se sabe que un $9,16 \%$ de la población española mayor de 18 años presenta algún grado de enfermedad renal crónica 3 .

Al finalizar 2010, más de 46.000 pacientes en España estaban en tratamiento sustitutivo de la función renal, siendo la hemodiálisis (HD) el tratamiento más prevalente $(45,5 \%)$ después del trasplante renal $(49,4 \%)$. Sin embargo, de los que comenzaron tratamiento renal sustitutivo durante el 2010 , un $80,8 \%$ lo hicieron en $H D$, un $13,3 \%$ en diálisis peritoneal y un $3 \%$ con trasplante renal ${ }^{4}$; este crecimiento supera con creces al de la oferta de órganos para trasplante y por tanto muchos pacientes deberán hemodializarse durante largos periodos de tiempo. Además, el envejecimiento de la población hace que cada vez lleguen a HD pacientes con mayor comorbilidad asociada.

Para poder llevar a cabo el tratamiento con HD es condición sine qua non disponer de un acceso vascular (AV) normofuncionante, dispositivo que permite poner en comunicación la sangre del paciente con un circuito externo que se encarga de su depuración.

La importancia del $\mathrm{AV}$ radica en que va a determinar la calidad de la HD recibida, lo que va a repercutir directamente en la calidad de vida del paciente ${ }^{5}$. 
El AV ideal debe reunir al menos tres requisitos: permitir un abordaje seguro y continuado del sistema vascular, proporcionar flujos suficientes para suministrar la dosis de HD programada y carecer de complicaciones. Actualmente no existe dicho $\mathrm{AV}$, sin embargo se considera la fístula arteriovenosa interna (FAVI) como el AV de elección debido a su gran supervivencia y menor número de complicaciones en comparación con el catéter venoso central (CVC), considerando éste último como AV únicamente cuando no es posible conseguir una fístula normofuncionante ${ }^{5-8}$.

Las FAVI pueden ser autólogas o protésicas (también Ilamadas injertos, que utilizan una prótesis de politetrafluoretileno - PTFE - con forma tubular). Entre estos dos tipos, las FAVI autólogas (y especialmente las radiocefálicas) son consideradas de elección porque presentan 10 veces menos complicaciones que las protési$\operatorname{cas}^{9,10,11,23}$. Sin embargo, no siempre es posible realizar una FAVI autóloga por depender del estado previo de los vasos sanguíneos del paciente.

Se dice que una FAVI presenta disfunción cuando se produce cualquier clase de complicación que la impide funcionar con normalidad. Las complicaciones más frecuentes son las estenosis (disminución de la luz del vaso en un punto o tramo, que dificulta el flujo de sangre) y las trombosis (oclusión total o parcial de la luz del vaso causada por un trombo; es la primera causa de pérdida del AV), susceptibles de ser reparadas mediante algún tipo de técnica radiológica intervencionista o quirúrgi$\mathrm{ca}^{6,11,12}$. La ventaja de la radiología intervencionista es que utiliza técnicas menos invasivas y que no consume el limitado capital venoso del paciente renal ${ }^{6,12}$.

Se han reportado múltiples causas que pueden llevar a un paciente con FAVI a pasar por la sala de Radiología Vascular Intervencionista como consecuencia de una estenosis o una trombosis ${ }^{6,14,24}$. Las técnicas terapéuticas de Radiología Vascular Intervencionista más frecuentes para las estenosis y las trombosis se denominan angioplastia transluminal percutánea 0 ATP (dilatación del área estenótica mediante la introducción de un catéter con balón que se infla a alta presión en ese área) y trombectomía (eliminación del trombo que impide el flujo de sangre en la FAVI), respectivamente. Sin embargo, en la práctica suele suceder que en muchas ocasiones las trombosis son consecuencia de una estenosis previa en el acceso, con lo que se realizan las dos técnicas terapéuticas en el mismo procedimiento.
Desgraciadamente el AV continúa representando el talón de Aquiles de la HD, estableciéndose como uno de los problemas de mayor comorbilidad y siendo la primera causa de ingresos hospitalarios de estos pacientes $24,8 \%$ según el estudio DOPPS ${ }^{13}$ - , con el consiguiente encarecimiento de los costes asociados al tratamiento con $\mathrm{HD}^{1,6}$.

El objetivo de este estudio fue detectar aquellos factores de riesgo más frecuentes que influyen en la disfunción de una FAVI, con el fin de minimizar las complicaciones estenóticas o trombóticas relacionadas con el AV y de esta forma disminuir la elevada morbimortalidad y costes asociados a las complicaciones del AV.

\section{Material y métodos}

Se trata de un diseño de casos y controles en el que los casos son los pacientes portadores de FAVI que han sufrido una disfunción estenótica o trombótica del AV $y$, los controles, son los pacientes portadores de FAVI que nunca han sufrido estenosis o trombosis de su AV. Por tanto, la variable principal para la inclusión en uno u otro grupo, fue el haberse realizado o no algún procedimiento terapéutico de radiología vascular intervencionista (ATP o trombectomía) sobre la FAVI a consecuencia de una disfunción 0 , por otro lado, el haberse realizado una segunda FAVI a causa de la trombosis de la primera FAVI, sin reparación radiológica intervencionista previa (algunas trombosis de larga evolución no son candidatas a reparación).

Previa autorización por parte del Comité Ético de Investigación Clínica de la Fundación Jiménez Díaz (FJD), este estudio se llevó a cabo en dicho centro, por tanto el ámbito del estudio lo constituye este hospital terciario de referencia para toda la población de la zona centro de Madrid, que atiende un área de $\mathbf{4 2 5 . 0 0 0}$ habitantes. El periodo para realizar la revisión de historias clínicas comprendió desde febrero de 2006 (año en que comenzó a funcionar la base de datos Indra utilizada en el hospital para la informatización de las historias clínicas) hasta marzo de 2012.

Los criterios de selección fueron: ser mayor de 18 años, haberse realizado la/s FAV en la Fundación Jiménez Díaz entre febrero de 2006 y enero de 2012, no haber tenido FAVI previa a febrero de 2006, y disponer de un seguimiento del paciente registrado en la base de datos del centro de al menos 2 meses para valorar su 
evolución. En 2 meses la FAVI ya se debería encontrar en condiciones de ser utilizada desde su realización y, en dicho periodo de tiempo, también es susceptible de sufrir alguna de las disfunciones señaladas. Por tanto, se incluyeron las FAVI con una antigüedad entre 2 meses y 6años.

Inicialmente se partió de una población diana de 272 pacientes con FAVI realizada entre febrero de 2006 y enero de 2012 aunque, tras la aplicación de los criterios de selección, resultó una población a estudio de 188 pacientes, incluyéndose a todos ellos como muestra con el fin de aumentar la potencia del estudio.

Se utilizó el programa estadístico SPSS 15.0 para Windows $^{\circledR}$, donde se registraron todas las variables de los pacientes referenciadas en la literatura, que pudieran estar relacionadas con la disfunción de una FAVI.

Las variables estudiadas se han dividido en tres grupos:

1. Características basales: edad, sexo y factores de riesgo cardiovascular diagnosticados en la historia clínica como: obesidad (representada por un índice de masa corporal -IMC- mayor a $24 \mathrm{~kg} / \mathrm{m}^{2}$ ), hipertensión arterial (HTA), diabetes mellitus (DM), dislipemia, tabaquismo (considerando factor de riesgo tanto el fumar como el haber fumado).

2. Variables clásicas relacionadas con la ERC: etiología de la enfermedad renal crónica; comienzo programado en HD (si la FAVI ha sido realizada antes de comenzar tratamiento con HD y no han sido necesarios CVC previos); tipo de FAVI y su localización; estado previo de los vasos y posibles lesiones en la capa íntima del sistema venoso del paciente (se revisó el número de días de hospitalización en la FJD desde febrero de 2006 hasta la fecha de realización de la FAVI, en los que se hubiera podido colocar algún tipo de dispositivo intravenoso, susceptible de provocar lesiones en la íntima del vaso sanguíneo); causa de disfunción del AV (estenosis, trombosis -si no se ha logrado solucionar la disfunción-, o ambassi la estenosis ha originado la trombosis y se resuelven ambas-); calibre previo de arteria y vena medido con eco-Doppler.

3. Otras variables relacionadas con la disfunción de la FAVI: número de sesiones de HD a la semana; calibre de la aguja utilizada (se asume como calibre estándar 15 Gauges); portar o haber portado catéteres venosos centrales, su tipo, localización y duración; ser portador de marcapasos; parámetros analíticos (hemoglobina - $\mathrm{Hb}$-, hormona paratiroidea -PTH-, producto calcio- fósforo -CaxP-) en el momento previo de la realización de ATP y/o trombectomía (en los pacientes que no han precisado técnicas radiológicas intervencionistas - grupo control - se tomaron los parámetros analíticos a los 2 meses de la realización de la FAVI); toma de medicación anticoagulante, antiagregante 0 ambas, estado cardiovascular (medido por la presencia de arteriosclerosis, cardiopatía isquémica y accidentes cerebrovasculares-ACV-) y cifras de presión arterial (considerando hipertenso si tiene cifras mayores a 140/90 $\mathrm{mmHg}$ e hipotenso si la presión arterial sistólica es menor de $85 \mathrm{mmHg}$ ), todo ello también previo a la realización de cada técnica radiológica intervencionista o a los 2 meses de la realización de la FAVI si no ha tenido lugar en el paciente una ATP y/o trombectomía; época del año más frecuente de realización de ATP y/o trombectomías.

Como variables confusoras cabe señalar los problemas en la maduración del acceso, problemas en cuanto a su punción, el grado de movilidad del paciente y el nivel de autocuidados de la FAVI, puesto que no ha sido posible objetivar su medida debido al carácter retrospectivo del estudio.

Otra posible limitación puede ser el periodo de tiempo estimado en los criterios de selección, ya que hubiera sido interesante poder estudiar también las FAVI funcionantes en la actualidad realizadas antes del año 2006, que han presentado o no disfunción en alguna ocasión, pero debido a la falta de datos objetivos antes de febrero de 2006, se decidió su exclusión del estudio para evitar sesgar los resultados.

Tras la revisión de las 188 historias clínicas, se realizó una descripción de los pacientes de forma global y estratificando por sexo y grupos de edad ( $<65$ años, $65-75$ años y $>75$ años) y realización o no de ATP (variable principal). La descripción de las variables cuantitativas se realizó mediante la media y la desviación estándar, para aquellas que seguían una distribución normal, o mediante la mediana y los cuartiles, para aquellas que no la seguían. La descripción de las variables cualitativas se realizó mediante tablas de frecuencias y tablas de contingencia.

Se evaluó la asociación de cada una de las variables con el evento de interés (haberse realizado o no ATP y/o trombectomía) utilizando para ello modelos de re- 
gresión logística univariable. No fue posible realizar un análisis multivariable posterior por falta de potencia estadística.

La significación estadística de las variables se evaluó a través del test de razón de verosimilitudes, tomándose un nivel de significación de 0,05 y una potencia del $80 \%$.

\section{Resultados}

\section{Características basales:}

Se ha obtenido globalmente que la media de edad de las personas que comienzan tratamiento renal sustitutivo con HD en la FJD desde febrero de 2006 a marzo de 2012 es de 64,28 $\pm 15,79$ años, habiendo un 56,9\% de hombres. La media del IMC es de $25,31 \pm 5,09 \mathrm{~kg} / \mathrm{m}^{2}$. La prevalencia de hipertensión se sitúa en el $84 \%$, siendo mayor que la de DM $(39,4 \%)$, dislipemia $(66,1 \%)$ y tabaquismo $(47,7 \%)$.

Atendiendo a la estratificación por sexo, la media de edad de las mujeres que inician HD es de $64,3 \pm 17,04$ años, siendo de $64,26 \pm 14,83$ años la de los hombres. Igualmente la media del IMC en las mujeres $(25,68 \pm$ $\left.6,53 \mathrm{~kg} / \mathrm{m}^{2}\right)$ es mayor que la de los hombres $(25,01 \pm$ $\left.3,49 \mathrm{~kg} / \mathrm{m}^{2}\right)$. Asimismo, la prevalencia de DM en mujeres $(44,4 \%)$ es mayor que en hombres $(35,5 \%)$. Por el contrario, la prevalencia de HTA es mayor en hombres ( $86 \%$ vs $81 \%$ ), así como la dislipemia $(69,2 \%$ vs $62 \%$ ) y el tabaquismo $(72,2 \%$ vs $16,9 \%)$.

En cuanto a la estratificación por edad, vemos que la frecuencia de HTA, DM y dislipemia aumenta con la edad hasta un $12 \%$, y que por el contrario se reduce el consumo de tabaco hasta en un $6 \%$.

Estratificando por el evento de interés (realización o no de ATP/trombectomía), obtenemos que la media de edad de los pacientes que se realizan ATP es de 65,13 $\pm 16,3$ años, siendo de $63,67 \pm 15,47$ años en aquellos que no han sufrido la realización de ATP. La media del IMC es muy similar en ambos grupos, siendo de 25,20 $\pm 4,67 \mathrm{~kg} / \mathrm{m}^{2}$ en el grupo ATP, y de 25,39 $\pm 5,4 \mathrm{~kg} /$ $\mathrm{m}^{2}$ en el otro grupo. Asimismo la frecuencia de ATP en hipertensos es del $35,44 \%$, en diabéticos del $29,7 \%$, en dislipémicos del $30,1 \%$ y en fumadores del $32,5 \%$.

En cuanto a los resultados de la regresión logística univariable que se pueden ver en la tabla 1 , sólo se encontró asociación estadísticamente significativa en la dislipemia. Por tanto se puede decir que con una seguridad del $95 \%$, los pacientes con dislipemia presentan un $60,9 \%$ menos de riesgo de presentar disfunción de la FAVI ( $p$-valor $=0,0031 ;$ OR: 0,391; IC: 0,208-0,730)

\begin{tabular}{|l|c|c|c|c|}
\hline Variable & OR & Error & [Int. conf. 95\%] & Valor p \\
\hline IMC & 0,993 & 0,0390 & $0,9171,072$ & 0,8496 \\
\hline Hipertensión & 0,627 & 0,2522 & $0,2851.393$ & 0,2489 \\
\hline Diabetes & 0,582 & 0,1846 & $0,3091,075$ & 0,0842 \\
\hline Dislipemia & 0,391 & 0,1251 & $0,2080,730$ & 0,0031 \\
\hline Fumador & 0,615 & 0,1939 & $0,3291,136$ & 0,1210 \\
\hline Sexo & 0,985 & 0,3003 & $0,5401,789$ & 0,9612 \\
\hline
\end{tabular}

Tabla 1. Influencia de las características basales en la disfunción de la fístula.

\section{Variables clásicas relacionadas con la ERC:}

La etiología de ERC más prevalente en la muestra estudiada es la nefropatía diabética seguida de la causa vascular.

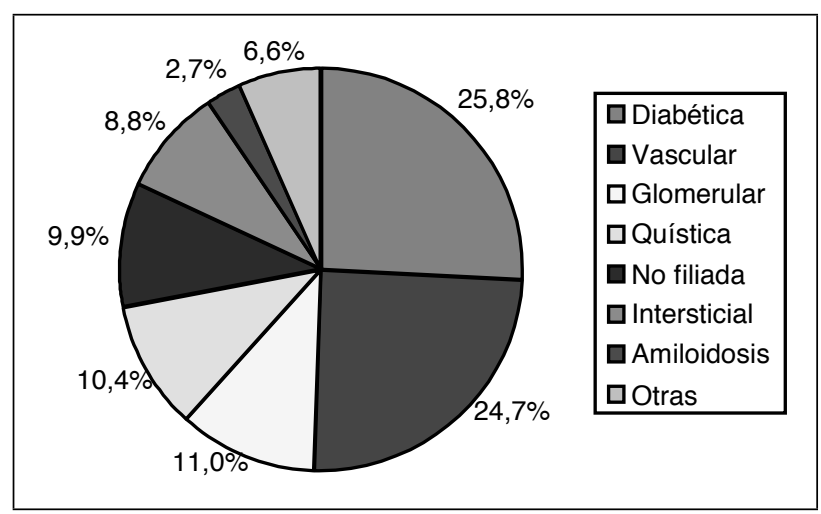

Figura 1. Etiología de la nefropatía.

En la descripción global se observa que el comienzo programado de la HD se produce en el $58 \%$ de los pacientes, es decir, inician el tratamiento con la FAVI como primer AV, sin CVC. Como primera causa de disfunción del AV resulta la estenosis (56,5\%), seguida de las trombosis causadas por las propias estenosis $(34,8 \%)$. En cuanto al tipo de FAVI, el $81,9 \%$ de las primeras FAVI realizadas son autólogas, disminuyendo al 42,5\% en la segunda FAVI realizada; a partir de la tercera FAVI todas ellas son protésicas. En cuanto a la localización de la primera FAVI, el 44,7\% son braquiocefálicas, seguidas por el $36,2 \%$ de radiocefálicas; ya en la segunda FAVI la localización va siendo más proximal con un $35 \%$ de braquiocefálicas, un 35\% de braquioaxilares y un $15 \%$ de braquiobasílicas; por último, en la construcción de las sucesivas FAVI, entre el $80 \%$ y el $100 \%$ son braquioaxilares. En cuanto al miembro superior donde se realizó la FAVI, el $79,3 \%$ de las primeras FAVI se 
realizaron en el brazo no dominante, cambiando en la segunda FAVI al brazo dominante en el $62,5 \%$ de los casos. Todas las arterias examinadas previamente a la realización de la FAVI tienen un calibre adecuado y, en cuanto al calibre de la vena, el $88,6 \%$ posee el calibre mínimo recomendado ( $3 \mathrm{~mm}$ o superior).

En cuanto a la estratificación por sexo, la FAVI autóloga es más frecuente en hombres $(87,9 \%$ vs $74,1 \%)$, así como la realización de la primera FAVI en el brazo no dominante $(83,2 \%$ vs $74,1 \%)$. También la localización radiocefálica (de elección) de la primera FAVI es más frecuente en hombres ( $43 \%$ vs $27,2 \%$ ). Por último, el $63,6 \%$ de los hombres comienzan HD de manera programada a diferencia de las mujeres que lo hacen en un $52 \%$.

La estratificación por grupos de edad se muestra en la tabla 2.

\begin{tabular}{|l|c|c|c|c|c|c|c|}
\hline & \multicolumn{3}{|c|}{$<65$} & \multicolumn{2}{c|}{$65-75$} & \multicolumn{2}{c|}{$>75$} \\
\hline Variable & N & $\%$ & N & $\%$ & N & $\%$ \\
\hline Tipo de 1 & FAVI & 65 & 85,5 & 36 & 81,8 & 53 & 77,9 \\
\hline autóloga & 11 & 14,5 & 8 & 18,2 & 15 & 22,1 \\
\hline PTFE & & & & & \\
\hline Causa 1 & a disfunción & 12 & 42,9 & 7 & 53,8 & 13 & 52,0 \\
\hline Estenosis & 7 & 25,0 & 3 & 23,1 & 9 & 36,0 \\
\hline Trombosis & 9 & 32,1 & 3 & 23,1 & 3 & 12,0 \\
\hline Ambas & & & & & \\
\hline Localización 1 & FAVI & 45 & 59,2 & 12 & 27,3 & 11 & 16,2 \\
\hline Radiocefálica & 0 & 0,0 & 1 & 2,3 & 1 & 1,5 \\
\hline Humerocefálica & 20 & 26,3 & 21 & 47,7 & 43 & 63,2 \\
\hline Braquiocefálica & 0 & 0,0 & 1 & 2,3 & 0 & 0,0 \\
\hline Humerobasílica & 5 & 6,6 & 4 & 9,1 & 3 & 4,4 \\
\hline Braquiobasílica & 1 & 1,3 & 1 & 2,3 & 1 & 1,5 \\
\hline Humeroaxilar & 5 & 6,6 & 4 & 9,1 & 9 & 13,2 \\
\hline Braquioaxilar & & & & \\
\hline
\end{tabular}

Tabla 2. Características de la $1^{\text {a }}$ FAVI realizada en función del grupo de edad.

Estratificando por el evento de interés (realización o no de ATP/trombectomía) y teniendo en cuenta la regresión logística de la tabla 3, se observa que:

- El $42,2 \%$ de los que están en tratamiento con HD han sufrido alguna disfunción de la FAVI, lo que contrasta con el $14,7 \%$ de los que aún no han co- menzado y han sufrido disfunción de su AV (p-valor=0,0015; 0R: 4,236; IC: 1,681-12,971).

- Entre las primeras FAVI realizadas, el 33,8\% han sufrido disfunción si eran autólogas y el $52,9 \%$ si eran protésicas ( $p$-valor $=0,0391$; 0R: 2,207; IC: 1,041- 4,724).

- El $30,9 \%$ de las FAVI radiocefálicas han sufrido disfunción, siendo en el resto de localizaciones de FAVI este porcentaje del $40,8 \%(p>0,05)$.

- En cuanto al calibre de la vena previo a la realización de la primera FAVI, era de $3,61 \mathrm{~mm}$ de media en el grupo que presentó disfunción, y de 4,43 mm de media en el otro grupo $(p>0,05)$.

\begin{tabular}{|l|c|c|c|c|c|}
\hline Variable & OR & Error & \multicolumn{2}{|c|}{$\begin{array}{c}\text { [nt. conf. } \\
\mathbf{9 5 \%}\end{array}$} & $\begin{array}{c}\text { Valor } \\
\mathbf{p}\end{array}$ \\
\hline Comenzar HD & 4,236 & 2,1645 & 1,681 & 12,971 & $\mathbf{0 , 0 0 1 5}$ \\
\hline Tipo de $1^{\text {a }}$ FAVI & 2,207 & 0,8463 & 1,041 & 4,724 & $\mathbf{0 , 0 3 9 1}$ \\
\hline Brazo de $1^{\text {a }}$ FAVI & 0,936 & 0,3467 & 0,456 & 1,966 & 0,8589 \\
\hline $\begin{array}{l}\text { Comienzo } \\
\text { programado }\end{array}$ & 0,766 & 0,2544 & 0,397 & 1,464 & 0,4205 \\
\hline $\begin{array}{l}\text { Localización } 1^{\text {a }} \\
\text { FAVI }\end{array}$ & 1,545 & 0,4967 & 0,829 & 2,936 & 0,1723 \\
\hline $\begin{array}{l}\text { Calibre vena previo } \\
\text { a 1 } 1^{a} \text { FAVI }\end{array}$ & 0,556 & 0,2280 & 0,227 & 1,055 & 0,0808 \\
\hline $\begin{array}{l}\text { Días ingreso previo } \\
\text { a 1 } 1^{a} \text { FAVI }\end{array}$ & 1,002 & 0,0059 & 0,990 & 1,014 & 0,7032 \\
\hline
\end{tabular}

Tabla 3. Influencia de las variables clásicas de ERC en la disfunción de la fístula.

(Categorías de referencia de las variables categóricas: comienzo $\mathrm{HD}=$ no; tipo de $1^{\text {a }} \mathrm{FAVI}=$ autóloga; brazo de $1^{\text {a }} \mathrm{FAVI}=$ miembro dominante; comienzo programado=no; localización $1^{\text {a }} \mathrm{FAVI}=$ radiocefálica)

\section{Otras variables relacionadas con la disfunción de la FAVI:}

Como se comentó anteriormente, 80 pacientes (43\%) sí han llevado un CVC yugular o subclavio previo a la realización de la primera FAVI. En concreto, 61 pacientes $(32,8 \%)$ han Ilevado un CVC y 19 pacientes $(9,7 \%)$ han Ilevado hasta dos CVC antes de su primera FAVI. EI 90,1\% de los pacientes a los que se implantó un CVC, éste fue tunelizado, localizándose la mayoría $(93,8 \%)$ en la vena yugular interna derecha.

Con la primera FAVI, 88 pacientes $(48,4 \%)$ no tomaban ningún tipo de antiagregante 0 anticoagulante, 72 pacientes $(39,6 \%)$ tomaban antiagregantes, 15 pacientes $(8,2 \%)$ tomaban anticoagulantes y 7 pacientes $(3,8 \%)$ tomaban ambos medicamentos. En los pacientes que 
han sufrido sucesivas disfunciones del AV, aumentó el porcentaje de los que tomaban antiagregantes hasta el $71,4 \%$.

En cuanto al estado cardiovascular, era bueno en 79 pacientes $(44,4 \%)$, presentaban arteriosclerosis 43 $(24,2 \%)$, ACV $19(10,7 \%)$ y cardiopatía isquémica 33 pacientes $(18,5 \%)$.

Al estudiar las épocas del año en que se producen las disfunciones, en la época de primavera y otoño se da el $54 \%$ y en verano e invierno el $46 \%$, produciéndose tres picos que corresponden a los meses de abril, julio y noviembre, a pesar de lo cual no se hallaron diferencias significativas.

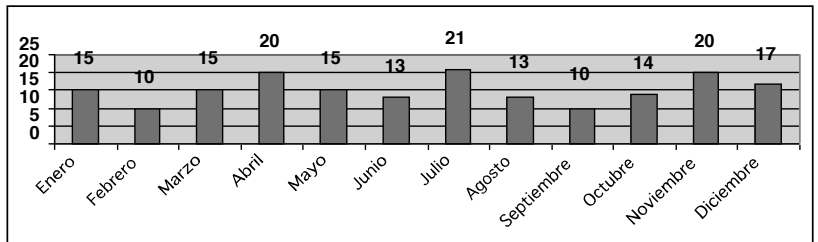

Figura 2. Distribución de todas las disfunciones registradas por meses.

En cuanto a la descripción de las variables cuantitativas estratificando por sexo, se aprecia que la media y mediana de los parámetros analíticos (PTH-que no sigue una distribución normal-, hemoglobina y producto calcio-fósforo) son ligeramente superiores en hombres que en mujeres.

En cuanto a la descripción de las características cualitativas también en función del sexo, es superior la incidencia de marcapasos en hombres $(6,6 \%$ vs $1,2 \%)$ aunque ocurre lo contrario con la incidencia de CVC previos, siendo superior en mujeres ( $48,8 \%$ vs $38,7 \%$ ). La toma de antiagregantes $\mathrm{y} / \mathrm{o}$ anticoagulantes es mayor en hombres $(58,8 \%$ vs $42,5 \%)$, a la vez que presentan peor estado cardiovascular que las mujeres $(66,3 \%$ vs $41,6 \%)$ con mayor prevalencia de arteriosclerosis y cardiopatía isquémica.

En la estratificación por edad, la media del producto calcio-fósforo es superior en la población más joven $(45,41 \pm 13,13$ en $<65$ años vs. $41,04 \pm 9,05$ en $>75$ años) al igual que ocurre con la mediana de las cifras de PTH (328 entre $162,25-529,4 \mathrm{pg} / \mathrm{ml}$ en $<65$ años vs. 251,5 entre $157,25-345 \mathrm{pg} / \mathrm{ml}$ en $>75$ años).

Estratificando por el evento de interés (realización o no de ATP/trombectomía por disfunción), se observa que a los pacientes que se les ha realizado ATP han portado
CVC previo durante más tiempo (mediana de 128 entre 89,5-125 días) que a los que no se les ha realizado ATP ( 118,5 entre 89,75-174 días). De igual manera, los pacientes que han sufrido disfunción de su FAVI han presentado mayores medias de hemoglobina $(11,62 \pm$ $1,38 \mathrm{~g} / \mathrm{dl}$ vs $11,29 \pm 1,57 \mathrm{~g} / \mathrm{dl}$ ) y producto calcio-fósforo $(43,87 \pm 10,82$ vs $42,1 \pm 11,99)$, y mayores medianas de PTH (368 entre $197,4-576,5 \mathrm{pg} / \mathrm{ml}$ vs 269,15 entre $155,7-381,75 \mathrm{pg} / \mathrm{ml})$.

En la tabla 4 se observa que el $45 \%$ de los que han tenido CVC previos presentan disfunción de la FAVI, siendo esta disfunción del $30 \%$ si no han portado nunca un CVC. Igualmente han sufrido disfunción el $75 \%$ de los pacientes que llevaron CVC en ambas yugulares (izquierda y derecha), siendo del $44 \%$ en los pacientes en los que la vena de localización del CVC fue la yugular interna derecha.

\begin{tabular}{|c|c|c|c|c|}
\hline & \multicolumn{2}{|c|}{ No ATP } & \multicolumn{2}{|c|}{ ATP } \\
\hline Variable & $\mathbf{N}$ & $\%$ & $\mathbf{N}$ & $\%$ \\
\hline \multicolumn{5}{|l|}{ CVC previos a la $1^{\text {a }}$ FAVI } \\
\hline No & 74 & 62,7 & 32 & 47,1 \\
\hline Si & 44 & 37,3 & 36 & 52,9 \\
\hline \multicolumn{5}{|c|}{ Número de CVC previos a la $1^{\mathrm{a}}$ FAVI } \\
\hline 0 & 74 & 62,7 & 32 & 47,1 \\
\hline 1 & 35 & 29,7 & 26 & 38,2 \\
\hline 2 & 9 & 7,6 & 9 & 13,2 \\
\hline 3 & 0 & 0,0 & 1 & 1,5 \\
\hline \multicolumn{5}{|c|}{ Localización de CVC previo/s a la $1^{\text {a }}$ FAVI } \\
\hline Yugular derecha & 42 & 95,5 & 33 & 91,7 \\
\hline $\begin{array}{l}\text { Ambas yugulares (izquierda y } \\
\text { derecha) }\end{array}$ & 1 & 2,3 & 3 & 8,3 \\
\hline Subclavia derecha & 1 & 2,3 & 0 & 0,0 \\
\hline
\end{tabular}

Tabla 4. Descripción de características cualitativas estratificando por ATP.

En cuanto a la regresión logística univariable, se puede ver en la tabla 5 que tanto la PTH en el momento de la primera ATP ( $p$-valor $=0,0188$ ), como el haber sido portador de CVC previo a la realización de la primera FAVI ( $p$-valor $=0,0381$ ), son variables asociadas a la disfunción de la FAVI. En concreto, haber sido portador de CVC antes de la realización de la FAVI aumenta casi al doble el riesgo de disfunción de la futura FAVI (OR: 1,892; IC: 1,036-3,482). 


\begin{tabular}{|c|c|c|c|c|c|}
\hline \multirow{2}{*}{$\begin{array}{l}\text { VARIABLE } \\
\text { Duración CVC previo/s } \\
\text { a } 1^{a} \text { FAVI (días) }\end{array}$} & \multirow{2}{*}{$\begin{array}{c}\text { OR } \\
1,001\end{array}$} & \multirow{2}{*}{\begin{tabular}{|l|} 
Error \\
0,0016
\end{tabular}} & \multicolumn{2}{|c|}{$\begin{array}{l}\text { [Int. conf. } \\
\text { 95\%] }\end{array}$} & \multirow{2}{*}{$\begin{array}{l}\text { Valor } \mathrm{p} \\
0,3504\end{array}$} \\
\hline & & & 0,998 & 1,005 & \\
\hline Hb previa a la $1^{a}$ ATP & 1,156 & 0,1186 & 0,948 & 1,420 & 0,1533 \\
\hline PTH previa a la $1^{\text {a }}$ ATP & 1,002 & 0,0007 & 1,000 & 1,003 & 0,0188 \\
\hline $\begin{array}{l}\text { Calcio previo a la } 1^{\mathrm{a}} \\
\text { ATP }\end{array}$ & 1,105 & 0,2266 & 0,741 & 1,663 & 0,6244 \\
\hline $\begin{array}{l}\text { Fósforo previo a la } 1^{a} \\
\text { ATP }\end{array}$ & 1,063 & 0,1152 & 0,857 & 1,316 & 0,5734 \\
\hline $\begin{array}{l}\text { CaxP previo a la } 1^{\mathrm{a}} \\
\text { ATP }\end{array}$ & 1,013 & 0,0137 & 0,987 & 1,041 & 0,3275 \\
\hline Marcapasos & 0,544 & 0,4521 & 0,078 & 2,439 & 0,4448 \\
\hline $\begin{array}{l}\text { Haber tenido CVC } \\
\text { previo a } 1^{\text {a }} \text { FAVI }\end{array}$ & 1,892 & 0,5840 & 1,036 & 3,482 & 0,0381 \\
\hline \multicolumn{6}{|c|}{ Número CVC previo/s a $1^{\text {a }}$ FAVI } \\
\hline 1 & 1,718 & 0,5744 & 0,891 & 3,317 & \\
\hline $2-3$ & 2,569 & 1,2997 & 0,950 & 7,062 & 0,0870 \\
\hline \multicolumn{6}{|c|}{ Toma de antiagreg. previos a $1^{\text {a }}$ ATP } \\
\hline Antiagregantes & 0,887 & 0,2937 & 0,461 & 1,695 & \\
\hline Anticoagulantes & 1,111 & 0,6347 & 0,345 & 3,366 & \\
\hline Ambos & 0,667 & 0,5768 & 0,092 & 3,288 & 0,9387 \\
\hline \multicolumn{6}{|c|}{ Estado cardiovascular previo a $1^{\mathrm{a}}$ ATP } \\
\hline Arteriosclerosis & 0,905 & 0,3469 & 0,424 & 1,914 & \\
\hline ACV & 0,580 & 0,3151 & 0,188 & 1,629 & \\
\hline IAM & 0,279 & 0,1411 & 0,096 & 0,713 & \\
\hline Ambos & 0,419 & 0,4931 & 0,020 & 3,434 & 0,0815 \\
\hline \multicolumn{6}{|c|}{ Presión arterial previa a $1^{\text {a }}$ ATP } \\
\hline Hipertenso & 0.965 & 0.3283 & 0.495 & 1.887 & \\
\hline
\end{tabular}

Tabla 5. Influencia de varias características sobre la disfunción de la fístula.

\section{Discusión}

\section{Características basales:}

A la vista de los resultados obtenidos, hay que tener en cuenta que tratamos con una población añosa (probablemente con una de las medias de edad poblacional más elevada del territorio español por ser hospital de referencia para toda la zona centro de Madrid) y pluripatológica, con alta prevalencia de factores de riesgo cardiovascular. El primer hallazgo significativo que aparece es la asociación entre presentar dislipemia y menor riesgo de disfunción de la FAVI: lo que inicialmente parece una contradicción ya que la dislipemia se plantea como factor de riesgo cardiovascular cobra sentido desde la crucial perspectiva nutricional, es decir, los niveles de lípidos suelen ser más elevados en pacientes con mejor estado nutricional porque comen más ${ }^{15,16}$, por ello se podría pensar que los pacientes con mejor estado nutricional presentarían menos riesgo de disfunción de su FAVI. Sería recomendable realizar futuros estudios sobre este tema.

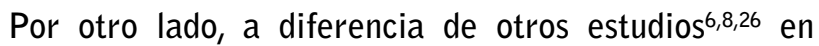
nuestra población las mujeres no presentan mayor riesgo de disfunción de la FAVI que los hombres, ni tampoco la edad juega un papel fundamental. Igualmente, a diferencia de algunas guías clínicas ${ }^{6}$ y estudios recientes como el de Bahadi y cols. ${ }^{25}$, en nuestra población no resultó significativa la diabetes como factor de riesgo en la disfunción de las FAVI.

\section{Variables clásicas relacionadas con la ERC:}

Conforme está escrito en la literatura ${ }^{6,16,21}$, la etiología de la ERC más prevalente en este estudio es la diabética y vascular.

A pesar de las recomendaciones de las guías nacionales e internacionales ${ }^{6,8,9,18}$ que aconsejan la remisión precoz del paciente al nefrólogo para enlentecer la progresión de la enfermedad y programar el AV si fuera necesario, se observa aún un alto porcentaje de pacientes que inician HD de forma urgente $(42 \%)$, habiendo diferencias entre hombres $(36,4 \%)$ y mujeres (48\%). Acorde con el estudio de Antón-Pérez y cols. ${ }^{17}$ resultó significativo que la colocación de CVC previos a la realización de la FAVI en nuestros pacientes, supone casi el doble de riesgo de presentar disfunción de esa futura FAVI, de ahí la importancia del inicio programado.

En nuestro medio observamos que las estenosis suponen el $91,3 \%$ de las causas de disfunción recuperables, y de ellas el $38,1 \%$ progresan a trombosis, por lo que no se deben perder de vista estudios como el de Gruss et al. sobre los programas de monitorización y seguimiento del acceso vascular ${ }^{19,20,28}$.

En cuanto al tipo de FAVI, tal y como señalan las guías clínicas $6,8,9$, se ha comprobado que como primera opción de AV se realizan FAVI autólogas y en el miembro superior no dominante, con localización radiocefálica en los pacientes más jóvenes y braquiocefálica en los más mayores. La elección de realizar una u otra está condicionado por el estado previo de los vasos del paciente y también en parte, por la menor dificultad técnica que tienen las FAVI protésicas para los cirujanos vasculares $^{6}$. También parece que el sexo masculino presenta mayor frecuencia en este tipo de FAVI "de elección" (autóloga, radiocefálica y en brazo no dominante) que 
de FAVI protésicas, las cuales presentan un riesgo significativamente superior de presentar disfunción cuantificado en más del doble en comparación con las FAVI autólogas.

En cuanto al inicio de HD, se ha observado 4 veces más riesgo de presentar disfunción de FAVI en aqueIlos pacientes que están en tratamiento renal sustitutivo con HD que los que aún se encuentran en la etapa prediálisis, en los cuales es más difícil diagnosticar la disfunción al no tener un seguimiento de su AV tan exhaustivo como los que están en HD. También podrían influir las consecuencias que este tratamiento crónico (HD) tiene sobre el organismo como la respuesta inflamatoria, calcificaciones, punciones repetidas de la FAVI, etc. 6,27.

En relación a este punto se ha visto que entre los que presentan disfunción, estos mantienen cifras más elevadas de hemoglobina, producto calcio-fósforo y PTH, resultando únicamente significativa ésta última.

\section{Otras variables relacionadas con la disfunción de la FAVI:}

A pesar de la significación de la PTH, éste es un parámetro que debe tomarse con cautela por estar influenciado a su vez por gran cantidad de factores.

Durante el periodo de funcionamiento normal de la primera FAVI, casi la mitad de los pacientes no tomaban medicación antiagregante y/o anticoagulante $y$, tras sucesivas disfunciones de la FAVI, casi las tres cuartas partes de pacientes la tomaban; esto puede interpretarse como que se tomaba para prevenir disfunciones en pacientes reincidentes, como refuerza el resultado del meta-análisis de Coleman et al que concluye que los antiagregantes plaquetarios reducen la tasa de trombosis de las FAVI autólogas 22,0 como que presentan mayor disfunción aquellos con patologías para las cuales precisan tratamiento antiagregante $y / 0$ anticoagulante. En cualquier caso hay que tener en cuenta que en tan sólo el $55,6 \%$ se ha hallado un buen estado cardiovascular. En concreto, los hombres presentan peor estado cardiovascular con mayor presencia de arteriosclerosis y cardiopatía isquémica, además de mayor ingesta de antiagregantes y/o anticoagulantes. También presentan mayor frecuencia de marcapasos al contrario de las mujeres, que presentan mayor incidencia de CVC previos, factor que aumenta el riesgo de disfunción de la FAVI como se vio anteriormente.
Podemos concluir que los pacientes con dislipemia, los que portan una fístula autóloga y los que no han iniciado hemodiálisis o lo han hecho de manera programada, se han asociado a un menor riesgo de disfunción de su fístula, con todos los beneficios que ello conlleva sobre la morbilidad asociada al paciente con enfermedad renal crónica.

Sería interesante realizar más estudios, especialmente prospectivos, sobre la influencia de los parámetros nutricionales sobre el acceso vascular y sobre la supervivencia de cada fístula en función de determinadas variables.

\section{Agradecimientos}

Al Dr. González Parra y a D. Ignacio Mahillo, estadístico de la Fundación Jiménez Díaz, por ayudar con los cálculos estadísticos.

Recibido: 14 Mayo 2013

Revisado: 16 Mayo 2013

Modificado: 30 Mayo 2013

Aceptado: 1 junio 2013

\section{Bibliografía:}

1. Martín de Francisco AL, Aguilera L, Fuster V. Enfermedad cardiovascular, enfermedad renal y otras enfermedades crónicas. Es necesaria una intervención más temprana en la enfermedad renal crónica. Nefrología. 2009; 29(1):6-9.

2. Zhang QL, Rothenbacher D. Prevalence of chronic kidney disease in population- based studies: Systematic review. [Internet]. BMC Public Health; 2008 [cited 2012 Feb 29]. Disponibleenhttp:// www.biomedcentral.com/1471-2458/8/117.

3. Martin de Francisco AL, Piñera C, Gago M, Ruiz J, Robledo C, Arias M. Epidemiología de la enfermedad renal crónica en pacientes no nefrológicos. Nefrología. 2009; 29 (Sup. Ext. 5): 101-5.

4. Registro Español de Enfermos Renales [Internet]. Vitoria: REER; 2005 [actualizado 2012; citado 1 Mar 2012]. Disponible en: http://rsaracho.dyndns. org/REER2/prevalencia3.php?pasotipotto $=$. 
5. Hiremath S, Knoll G, Weinstein MC. Should the Arteriovenous Fistula Be Created before Starting Dialysis?: A Decision Analytic Approach. PloS ONE. 2011; 6 (12): e28453. doi:10.1371/journal. pone. 0028453 .

6. Rodríguez JA, Gutiérrez JM, coordinadores. Guía de Acceso Vascular en Hemodiálisis [Internet]. Barcelona: Sociedad Española de Nefrología; 2004 [actualizado 22 Nov 2004; citado 1 Mar 2012]. Disponible en : http://www.senefro.org/ modules/webstructure/files/guia_acceso_vascular. pdf?check_idfile $=984$.

7. Remón C, Bardón E, Vila ML. Accesos para comenzar depuración extrarrenal: vascular y peritoneal. Acceso temporal en prediálisis. Nefrología. 2008; 28 (Supl. 3): 105-12.

8. Fluck R, Kumwenda M. Clinical Practice Guidelines Vascular Access for Haemodialysis. [Internet]. Hampshire: UK Renal Association; 2011 [cited 2012 Mar 2]. Available from: http://www.renal. org/Libraries/Guidelines/Vascular_Access_for_ Haemodialysis_-_FINAL_VERSION_-_05_January_2011.sflb.ashx.

9. Clinical Practice Guidelines and Clinical Practice Recommendations, 2006 Updates: Hemodialysis Adequacy, Peritoneal Dialysis Adequacy, Vascular Access [Internet]. New York: National Kidney Foundation; 2006 [cited 2012 Mar 2]. Available from: http://www.kidney.org/professionals/KD0QI/ guideline_upHD_PD_VA/va_guide2.htm.

10. Jiménez P. Fístulas arteriovenosas para hemodiálisis. [Internet]. Barcelona: Nefrología al día; 2011 [actualizado 2 Feb 2011; citado 7 Mar 2012]. Disponible en: http://nefrologiadigital.revistanefrologia.com/modules. .php?name=libro\&op=viewCap\&i dpublication=1\&idedition=13\&idcapitulo=73.

11. Bent $C L$, Sahni VA, Matson MB. The radiological management of the thrombosed arteriovenous dialysis fistula. Clin Radiol. 2011; 66: 1-12.

12. García J, Lacasa N, Muray S, Pérez I, García V. Accesos vasculares para hemodiálisis trombosados: rescate mediante técnicas de radiología vascular intervencionista. Nefrología. 2009; 29 (3): 24955.
13. Rayner HC, Pisoni RL, Bommer J, Canaud B, Hecking $E$, Locatelli $F$, et al. Mortality and hospitalization in haemodialysis patients in five European countries: results from the Dialysis Outcome and Practice Patterns Study (DOPPS). Nephrol Dial Transplant. 2004; 19 (1): 108-20.

14. Barbosa F. Indicación del acceso vascular desde el punto de vista nefrológico. En: González MT, Martínez R. Manual de accesos vasculares para hemodiálisis. Barcelona: Marge Médica Books; 2010: 25-34.

15. Brown RO, Compher C. A.S.P.E.N. clinical guidelines: nutrition support in adult acute and chronic renal failure. JPEN J Parenter Enteral Nutr. 2010; 34 (4): 366-77.

16. de Francisco AL, Sanjuán $F$, Foraster $A$, Fabado $S$, Carretero D, Santamaría C, et al. Epidemiological study on chronic renal failure elderly patients on hemodialysis. Nefrología. 2008; 28 (1): 48-55.

17. Antón-Pérez G, Pérez-Borges $P$, Alonso-Almán $F$, Vega-Díaz N. Accesos vasculares en hemodiálisis: un reto por conseguir. Nefrología. 2012; 32 (1): 103-7.

18. KDIG0 Clinical Practice Guidelines for the Diagnosis, Evaluation, Prevention, and Treatment of Chronic Kidney Disease-Mineral and Bone Disorder (CKD- MBD) [Internet]. New York: Kidney disease: Improving Global Outcomes; 2008 [cited 2012 May 20]. Available from: http://www.kdigo. org/guidelines/mbd/index.html.

19. Gruss $E$, Portolés J, Jiménez $P$, Hernández $T$, Rueda JA, Cerro J, et al. Seguimiento prospectivo del acceso vascular en hemodiálisis mediante un equipo multidisciplinar. Nefrología. 2006; 26 (6): 703-10.

20. Scaffaro LA, Bettio JA, Cavazzola SA, Campos BT, Burmeister JE, Pereira RM, et al. Maintenance of hemodialysis arteriovenous fistulas by an interventional strategy: clinical and duplex ultrasonographic surveillance followed by transluminal angioplasty. J Ultrasound Med. 2009; 28 (9): 1159-65.

21. Pérez-García R, Martín-Malo $A$, Fort $J$, Cuevas $X$, Lladós F, Lozano J, et al. ANSWER study. Baseline characteristics of an incident haemodialysis popu- 
lation in Spain: results from ANSWER--a multicentre, prospective, observational cohort study. Nephrol Dial Transplant. 2009; 24 (2): 578-88.

22. Coleman CI, Tuttle LA, Teevan C, Baker WL, White CM, Reinhart KM. Antiplatelet agents for the prevention of arteriovenous fistula and graft thrombosis: a meta analysis. Int J Clin Pract. 2010; 64 (9): 1239-44.

23. Reinhold $C$, Haage $P$, Hollenbeck M, Mickley $V$, Ranft J. Multidisciplinary management of vascular access for haemodialysis: from the preparation of the initial access to the treatment of stenosis and thrombosis. Vasa. 2011; 40 (3):188-98.

24. Smith GE, Gohil R, Chetter IC. Factors affecting the patency of arteriovenous fistulas for dialysis access. J Vasc Surg. 2012; 55 (3): 849-55.
25. Bahadi A, Hamzi MA, Farouki MR, Montasser D, Zajjari Y, Arache W, et al. Predictors of early vascular-access failure in patients on hemodialysis. Saudi J Kidney Dis Transpl. 2012; 23 (1): 83-7.

26. Riera-Vázquez $R$, Cordobès-Gual $J$, Lozano-Vilardell P, Manuel-Rimbau E, Corominas-Roura C, Juliá-Montoya J. Selección del tipo de acceso vascular en pacientes crónicos y agudos. Angiología. 2005; 57 (Supl 2): 35-45.

27. Oliva JS, Roa LM, Lara A, Garrido S, Salgueira $M$, Palma $A$, et al. Survival and factors predicting mortality in hemodialysis patients over 75 years old. J Nephrol. Forthcoming 2012.

28. Andrés $M M$, Gruss $E$, Martínez $S$, Piña $D$, Gálvez $M C$, Gago MC, et al. Comparación de dos métodos de seguimiento de las fístulas arteriovenosas protésicas incidentes en un área de salud. Rev Soc Esp Enferm Nefrol. 2011; 14 (3): 163-6. 